\title{
Deterministic Online Call Control in Cellular Networks and Triangle-Free Cellular Networks
}

\author{
Joseph Wun-Tat Chan* Francis Y.L. Chin ${ }^{\dagger} \quad$ Xin $\operatorname{Han}^{\ddagger} \quad$ Ka-Cheong Lam ${ }^{\S}$ \\ Hing-Fung Ting $₫ \quad$ Yong Zhang\|
}

\begin{abstract}
Wireless Communication Networks based on Frequency Division Multiplexing (FDM in short) plays an important role in the field of communications, in which each request can be satisfied by assigning a frequency. To avoid interference, each assigned frequency must be different to the neighboring assigned frequencies. Since frequency is a scarce resource, the main problem in wireless networks is how to fully utilize the given bandwidth of frequencies. In this paper, we consider the online call control problem. Given a fixed bandwidth of frequencies and a sequence of communication requests arrive over time, each request must be either satisfied immediately after its arrival by assigning an available frequency, or rejected. The objective of call control problem is to maximize the number of accepted requests. We study the asymptotic performance of this problem, i.e., the number of requests in the sequence and the bandwidth of frequencies are very large. In this paper, we give a $7 / 3$ competitive algorithm for call control problem in cellular network, improving the previous 2.5-competitive result. Moreover, we investigate the triangle-free cellular network, propose a 9/4-competitive algorithm and prove that the lower bound of competitive ratio is at least $5 / 3$.
\end{abstract}

Keywords: Online algorithms, Call control problem, Cellular networks, Triangle-free cellular network

\section{Introduction}

Frequency Division Multiplexing (FDM in short) is commonly used in wireless communications. To implement FDM, the wireless network is partitioned into small regions (cell) and each cell is equipped with a base station. When a call request arrives at a cell, the base station in this cell will assign a frequency to this request, and the call is established via this frequency. Since frequency is a scarce resource, to satisfy the requests from many users, a straightforward idea is reusing the same frequency for different call requests. But if two calls which are close

\footnotetext{
${ }^{*}$ College of International Education, Hong Kong Baptist University, Hong Kong, cswtchan@gmail.com

${ }^{\dagger}$ Department of Computer Science, The University of Hong Kong, Hong Kong, chin@cs.hku.hk, Research supported by HK RGC grant HKU-7117/09E and the William M.W. Mong Engineering Research Fund

${ }^{\ddagger}$ School of Software, Dalian University of Technology, China, hanxin.mail@gmail.com. Partially supported by Start-up Funding (1600-893335) provided by DUT, China

${ }^{\S}$ College of Computer Science, Zhejiang University, China, pandaman@163.com

`Department of Computer Science, The University of Hong Kong, Hong Kong, hfting@cs.hku.hk, Research supported by HK RGC grant HKU-7171/08E

"Department of Computer Science, The University of Hong Kong, Hong Kong, yzhang@cs.hku.hk
} 
to each other are using the same frequency, interference will happen to violate the quality of communications. Thus, to avoid interference, the same frequency cannot be assigned to two different calls with distance close to each other. In general, the same frequency cannot be assigned to two calls in the same cell or neighboring cells.

There are two research directions on the fully utilization of the frequencies. One is frequency assignment problem, and the other is call control problem. In frequency assignment problem, each call request must be accepted, and the objective is to minimize the number of frequencies to satisfy all requests. In call control problem, the bandwidth of frequency is fixed, thus, when the number of call requests in a cell or in some neighboring cells is larger than the total bandwidth, the request sequence cannot be totally accepted, i.e., some requests would be rejected. The objective of call control problem is to accept the requests as many as possible.

\section{Problem Statement:}

In this paper, we consider the online version of call control problem. There are $\omega$ frequencies available in the wireless networks. A sequence $\sigma$ of call requests arrives over time, where $\sigma=\left\{r_{1}, r_{2}, \ldots, r_{t}, \ldots\right\}, r_{t}$ denotes the $t$-th call request and also represent the cell where the $t$-th request arrives. When a request arrives at a cell, the system must either choose a frequency to satisfy this request without interference with other assigned frequencies in this cell and its neighboring cells, or reject this request. When handling a request, the system does not know any information about future call requests. We assume that when a frequency is assigned to a call, this call will never terminate and the frequency cannot be changed. The objective of this problem is to maximize the number of accepted requests.

We focus on the call control problem in cellular networks and triangle-free cellular networks. In the cellular networks, each cell is a hexagonal region and has six neighbors, as shown in Figure 1(a). The cellular network is widely used in wireless communication networks. A network is triangle-free if there are no 3 -cliques in the network, i.e., there are no three mutually-adjacent cells. An example of a triangle-free cellular network is shown in Fig. 1(b).

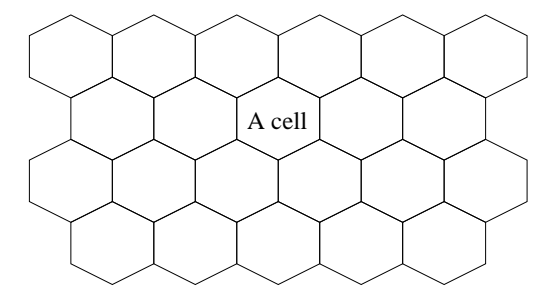

(a) cellular network

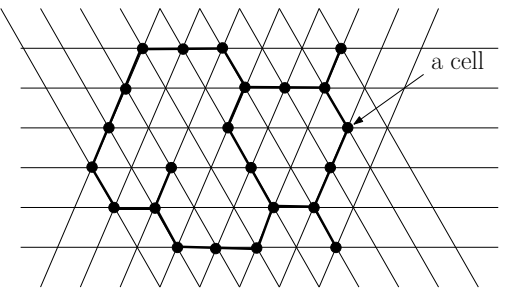

(b) triangle-free cellular network

Figure 1: An example of the cellular network and triangle-free cellular network

\section{Performance Measure:}

To measure the performance of online algorithms, we use the competitive ratio to compare the performance between the online algorithm and the optimal offline algorithm, which knows the whole request sequence in advance. In call control problem, the output is the number of accepted requests. For a request sequence $\sigma$, let $A(\sigma)$ and $O(\sigma)$ denote the number of accepted request of an online algorithm $A$ and the optimal offline algorithm $O$, respectively. We focus on the asymptotic performance for the call control problem, i.e., the number of requests and the number of frequencies are very large positive integers. The asymptotic competitive ratio for an 
online algorithm $A$ is

$$
R_{A}^{\infty}=\limsup _{n \rightarrow \infty} \max _{\sigma}\left\{\frac{O(\sigma)}{A(\sigma)} \mid O(\sigma)=n\right\}
$$

\section{Related Works:}

How to fully utilize the frequencies to satisfy the communication requests is a very fundamental problem in theoretical computer science and wireless communications. Both the frequency assignment problem and the call control problem are well studied during these years. From the description of these two problems, we know that the call control problem is the dual problem of the frequency assignment problem.

The offline version of the frequency assignment problem in cellular networks was proved to be NP-hard by McDiarmid and Reed [6], and two 4/3-approximation algorithms were given in $[6,7]$. In the online frequency assignment problem, when a call request arrives, the network must immediately assign a frequency to this call without any interference. There are mainly three strategies: Fixed Allocation [5], Greedy Assignment [1], and Hybrid Assignment [3]. If the duration of each call is infinity and the assigned frequency cannot be changed, the hybrid algorithm gave the best result for online frequency assignment, i.e., a 2-competitive algorithm for the absolute performance and a 1.9126-competitive algorithm for the asymptotic performance. When the background network is triangle-free, a 2-local 5/4-competitive algorithm was given in [9], an inductive proof for the $7 / 6$ ratio was reported in [4], where $k$-local means when assigning a frequency, the base station only knows the information of its neighboring cells within distance $k$. In [11], a 1-local 4/3-competitive algorithm was given.

For the call control problem, the offline version is NP-hard too [6]. To handle such problem, greedy strategy is always the first try, when a call request arrives, the network choose the minimal available frequency to serve this request, if any frequency is interfere with some neighboring assigned frequency, the request will be rejected. Pantziou et al. [8] analyzed the performance of the greedy strategy, proved that the asymptotic competitive ratio of the greedy strategy is equal to the maximal degree of the network. Caragiannis et al. [1] gave a randomized algorithm for the call control problem in cellular networks, the asymptotic competitive ratio of their algorithm is 2.651. Later, the performance of the randomized algorithms was improved to $16 / 7$ by the same authors [2], they also proved the lower bound of the asymptotic competitive ratio for the randomized algorithm is at least 2 . Very recently, a deterministic algorithm with asymptotic competitive ratio 2.5 was given in [10], and the lower bound of the asymptotic competitive ratio for the deterministic algorithm was proved to be 2 .

\section{Our Contributions:}

In this paper, we consider the deterministic algorithms for the online call control problem in cellular networks and triangle-free cellular networks. In cellular network, we give a $7 / 3$ competitive algorithm, improving the previous 2.5-competitive result. In triangle-free network, we propose a 9/4-competitive algorithm, moreover, we show that the lower bound of the competitive ratio in triangle-free network is at least $5 / 3$.

\section{Call Control in Cellular Networks}

The idea of our algorithm for call control problem in cellular networks is similar to the algorithm in [10]. By using a totally different analysis, the performance of our algorithm is better. Moreover, our algorithm is best possible among algorithms using this kind of idea. 
Cellular networks are 3 colorable, each cell can be associated with a color from $\{R, G, B\}$ and any two neighboring cells are with different colors. Partition the frequencies into four sets, $F_{R}, F_{B}, F_{G}$, and $F_{S}$, where $F_{X}(X \in\{R, G, B\})$ can be only used in cells with color $X$ and $F_{S}$ can be used in any cell. Since we consider the asymptotic performance of the call control problem, we may regard the number $\omega$ of frequencies in the system is a multiple of 7 . Divide the the frequencies into four disjoint sets as follows:

$$
\begin{aligned}
& F_{R}=\{1, \ldots, 2 \omega / 7\}, \\
& F_{G}=\{2 \omega / 7+1, \ldots, 4 \omega / 7\}, \\
& F_{B}=\{4 \omega / 7+1, \ldots, 6 \omega / 7\}, \text { and } \\
& F_{S}=\{6 \omega / 7+1, \ldots, \omega\}
\end{aligned}
$$

Obviously, the ratio between the number of frequencies in $F_{R}, F_{G}, F_{B}$, and $F_{S}$ is $2: 2: 2: 1$.

Now we describe our algorithm CACO as follows:

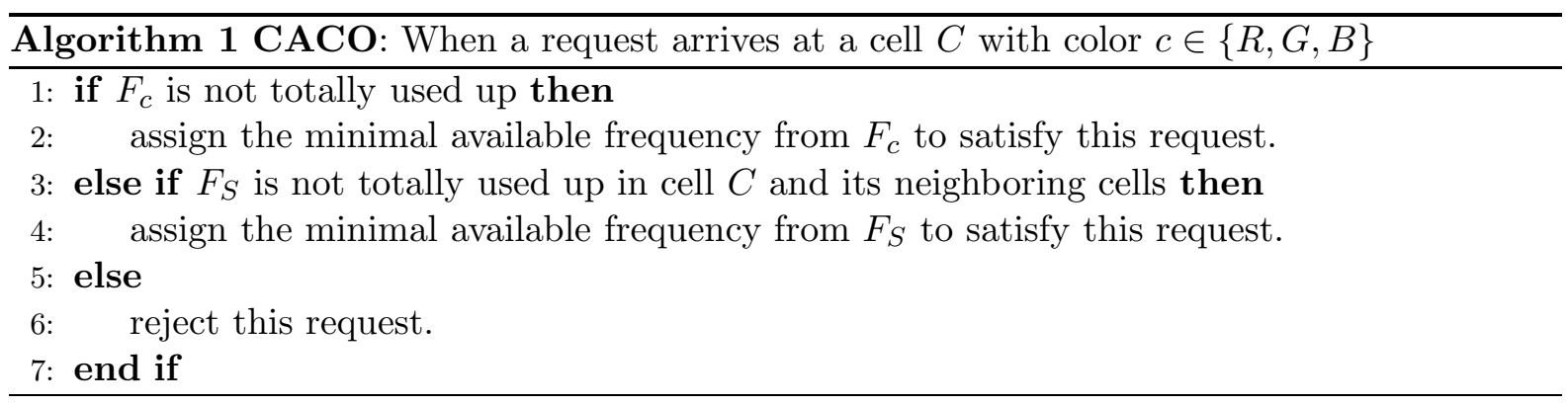

The high level idea to show the performance of our algorithm CACO is to prove that the ratio between the total number of accepted requests by $\mathrm{CACO}$ and the total number of satisfied requests by the optimal offline algorithm is at least $3 / 7$. To prove this, we analyze the number of satisfied requests in each cell and its neighboring cells, then compare the number with the optimum value.

Let $R_{i}$ be the number of the requests arrived in cell $C_{i}$. Let $O_{i}$ be the number of requests accepted by the optimal offline algorithm in cell $C_{i} . \quad \sum_{i} O_{i}$ is the total number of accepted request by the optimal offline algorithm. Let $A_{i}$ be the number of requests accepted by our online algorithm $\mathrm{CACO}$ in cell $C_{i} . \sum_{i} A_{i}$ is the total number of accepted request by CACO. Let $A_{x}\left(C_{i}\right)$ be the the number of requests accepted by $\mathrm{CACO}$ in cell $C_{i}$ by assigning frequencies from frequency set $F_{x}$. It can be seen that $A_{i}=A_{R}\left(C_{i}\right)+A_{G}\left(C_{i}\right)+A_{B}\left(C_{i}\right)+A_{S}\left(C_{i}\right)$. If $C_{i}$ is colored with $x \in\{R, G, B\}$, then $A_{i}=A_{x}\left(C_{i}\right)+A_{S}\left(C_{i}\right)$.

Fact 1. For each cell $C_{i}, O_{i} \leq R_{i}, A_{i} \leq R_{i}$, and $A_{i} \geq 2 \omega / 7$ when $R_{i} \geq 2 \omega / 7$.

According to the number of satisfied requests by the optimal offline algorithm, we classify the cells into two types: cell $C_{i}$ is safe if $O_{i} \leq 2 \omega / 3$, and dangerous otherwise.

Lemma 2. Suppose cell $C_{i}$ is with color $x$, if $C_{i}$ is safe, then $A_{i} \geq 3 O_{i} / 7$

Proof. Consider the following two cases.

- $R_{i} \leq 2 \omega / 7$

According to CACO, all requests in this cell must be satisfied when $R_{i} \leq 2 \omega / 7$, thus, $A_{i}=R_{i}$. Since $R_{i} \geq O_{i}$, we have $A_{i} \geq 3 O_{i} / 7$. 
- $R_{i}>2 \omega / 7$

In this case, $\mathrm{CACO}$ will accept at least $2 \omega / 7$ requests by assigning frequencies from $F_{x}$, thus, $A_{i} \geq 2 \omega / 7$. Since $C_{i}$ is safe, $O_{i} \leq 2 \omega / 3$, therefore, we have $A_{i} \geq 3 O_{i} / 7$.

Combining the above two cases, this lemma is true.

Fact 3. A safe cell has at most 3 dangerous neighboring cells. All neighboring cells around a dangerous cell are safe.

Proof. This fact can be proved by contradiction. If a safe cell $C$ has more than 3 dangerous neighboring cells, since $C$ has 6 neighboring cells, there must exist two dangerous cells which are neighbors. From the definition of dangerous cell, the total number of accepted request in these two dangerous neighboring cells is strictly more than $\omega$, contradiction!

Similarly, if a dangerous cell $C^{\prime}$ is a neighboring cell of another dangerous cell $C$, the total number of accepted request in $C$ and $C^{\prime}$ is strictly more than $\omega$. Contradiction!

According to the algorithm $\mathrm{CACO}$, when a request cannot be satisfied in a cell $C$ with color $c$, all frequencies in $F_{c}$ must be used in $C$, and all frequencies in $F_{S}$ must be used in $C$ and its six neighbors. Thus, we have the following fact:

Fact 4. If cell $C$ cannot satisfy a request according to the algorithm $C A C O$, then $A_{S}(C)+$ $\sum_{k} A_{S}\left(C_{k}\right) \geq \omega / 7$, where $C_{k}$ represents the neighboring cell of $C$.

To compare the number of satisfied requests by $\mathrm{CACO}$ in each cell with the optimal offline solution, we define $B_{i}$ as follows, where $C_{k}$ represents the neighboring cell of $C_{i}$.

$$
B_{i}= \begin{cases}3 O_{i} / 7 & \text { if } C_{i} \text { is safe } \\ A_{i}+\sum_{k}\left(A_{k}-3 O_{k} / 7\right) / 3 & \text { if } C_{i} \text { is dangerous. }\end{cases}
$$

Lemma 5. $\sum_{i} B_{i} \leq \sum_{i} A_{i}$.

Proof. Suppose $C_{k}$ is a safe cell. According to Lemma 2, we have $A_{k} \geq 3 O_{k} / 7$. From Fact 3, we know that there are at most three dangerous neighbors around $C_{k}$, thus, after counting $B_{k}=$ $3 O_{k} / 7$ frequencies in $C_{k}$, the remaining $A_{k}-3 O_{k} / 7$ frequencies can compensate the frequencies in its dangerous neighbors, and each dangerous cell receives $\left(A_{k}-3 O_{k} / 7\right) / 3$ frequencies. From the definition of $B_{i}$, we can see that $\sum_{i} B_{i} \leq \sum_{i} A_{i}$.

Theorem 6. The asymptotic competitive ratio of algorithm CACO is at most 7/3.

Proof. From the definition of $O_{i}$ and $B_{i}$, we can say that $O_{i} / B_{i} \leq 7 / 3$ for any cell $C_{i}$ leads to the correctness of this theorem. That is because

$$
\frac{\sum_{i} O_{i}}{\sum_{i} A_{i}} \leq \frac{\sum_{i} O_{i}}{\sum_{i} B_{i}} \leq \max _{i} \frac{O_{i}}{B_{i}}
$$

If the cell $C_{i}$ is safe, i.e., $O_{i} \leq 2 \omega / 3$, we have $O_{i} / B_{i}=7 / 3$.

If the cell $C_{i}$ is dangerous, i.e., $O_{i}>2 \omega / 3$, since $R_{i} \geq O_{i}>2 \omega / 3>3 \omega / 7$, that means the number of requests $R_{i}$ in this cell is larger than $A_{i}$. Thus, some requests are rejected, and this cell cannot accept any further requests. 
- If the number of accepted requests in any neighbor of $C_{i}$ is no more than $2 \omega / 7$, that means all frequencies in $F_{S}$ are assigned to requests in cell $C_{i}$. Thus, $A_{i}=3 \omega / 7$. In this case, we have

$$
O_{i} / B_{i}=O_{i} /\left(A_{i}+\left(\sum_{k}\left(A_{k}-3 O_{k} / 7\right)\right) / 3\right) \leq O_{i} / A_{i} \leq \omega / A_{i}=7 / 3
$$

- Otherwise, suppose there are $m$ neighbors of $C_{i}$ in which the number of accepted requests are more than $2 \omega / 7$. Let $\hat{O}_{i}$ denote the average number of the optimum value of accepted requests in these $m$ neighboring cells around $C_{i}$.

$$
\begin{aligned}
B_{i} & =A_{i}+\left(\sum_{k}\left(A_{k}-3 O_{k} / 7\right)\right) / 3 \\
& =2 \omega / 7+A_{S}\left(C_{i}\right)+\left(\sum_{k}\left(A_{k}-3 O_{k} / 7\right)\right) / 3 \\
& \geq 2 \omega / 7+A_{S}\left(C_{i}\right)+\left(m \times 2 \omega / 7+\sum_{\begin{array}{c}
\text { for the neighbors } \\
\text { with } A_{k}>2 \omega / 7
\end{array}} A_{S}\left(C_{k}\right)-m \times 3 \hat{O}_{i} / 7\right) / 3 \\
& \geq 2 \omega / 7+\left(m \times 2 \omega / 7+\sum_{\begin{array}{c}
\text { for the neighbors } \\
\text { with } A_{k}>2 \omega / 7
\end{array}} A_{S}\left(C_{k}\right)+A_{S}\left(C_{i}\right)-m \times 3 \hat{O}_{i} / 7\right) / 3 \\
& \geq 2 \omega / 7+\left(m \times 2 \omega / 7+\omega / 7-m \times 3 \hat{O}_{i} / 7\right) / 3 \\
& \geq 2 \omega / 7+\left(2 \omega / 7+\omega / 7-3 \hat{O}_{i} / 7\right) / 3
\end{aligned}
$$

(that is because for any neighbor with $A_{k}>2 w / 7$,

$O_{k} \leq\left(\omega-O_{i}\right) \leq \omega / 3$, thus, $\hat{O}_{i} \leq \omega / 3$ and $2 \omega / 7-3 \hat{O}_{i} / 7 \geq 0$.)

$\geq 2 \omega / 7+\left(3 \omega / 7-3\left(\omega-O_{i}\right) / 7\right) / 3$

(since $O_{k} \leq \omega-O_{i}$, we have $\hat{O}_{i} \leq \omega-O_{i}$ )

$=2 \omega / 7+O_{i} / 7$

Thus, $O_{i} / B_{i} \leq O_{i} /\left(2 w / 7+O_{i} / 7\right) \leq 7 / 3$.

From the above analysis, we can say that the asymptotic competitive ratio of the algorithm $\mathrm{CACO}$ is at most $7 / 3$.

In this kind of algorithms, the frequencies are partitioned into $F_{R}, F_{G}, F_{B}$ and $F_{S}$, when a request arrives at a cell with color $c$, first choose the frequency from the set $F_{c}$, then from $F_{S}$ if no interference appear. The performances are different w.r.t. the ratio between $\left|F_{R}\right|\left(\left|F_{G}\right|,\left|F_{B}\right|\right)$ and $\left|F_{S}\right|$. Note that from symmetry, the size of $F_{R}, F_{G}$ and $F_{B}$ should be same. Now we show that $\mathrm{CACO}$ is best possible among such kind of algorithms. Suppose the ratio between $\left|F_{R}\right|$ and $\left|F_{S}\right|$ is $x: y$. Consider the configuration shown in Figure 2. In the first step, $\omega$ requests arrive at the center cell $C$ with color $c$, the algorithm will use up all frequencies in $F_{c}$ and $F_{S}$, in this case, the ratio of accepted requests by the optimal offline algorithm and the online algorithm is $(3 x+y) /(x+y)$ since the optimal algorithm will accept all these requests. In the second step, $\omega$ requests arrive at $C_{1}, C_{2}$ and $C_{3}$ with the same color $c^{\prime}$. The online algorithm can only accept $x \omega /(3 x+y)$ requests in each $C_{i}(1 \leq i \leq 3)$ since the frequencies in $F_{S}$ are all used in $C$. In this case, the ratio between the optimal offline algorithm and the online algorithm is 
$3(3 x+y) /(4 x+y)$ since the optimal algorithm will accept all $\omega$ requests in $C_{i}(1 \leq i \leq 3)$ and reject all requests in $C$. Balancing these two ratios, we have $x: y=2: 1$. From the description of the above two steps, the lower bound of competitive ratio for this kind of algorithm is $7 / 3$.

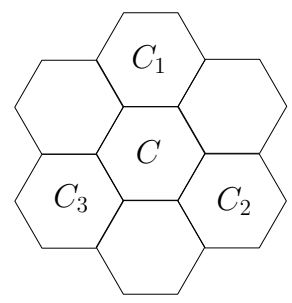

Figure 2: Algorithm CACO is best possible among this kind of algorithms

\section{Call Control in Triangle-Free Cellular Networks}

The call control problem in cellular network is hard. But for some various graph classes, this problem may have a better performance. For example, in linear network, an optimal online algorithm with competitive ratio $3 / 2$ can be achieved [10]. An interesting induced network, triangle-free cellular network, has been studied for many problems including frequency assignment problem $[4,9,11]$.

For a cell $C_{i}$ in triangle-free cellular networks, there are only two possible configurations for its neighboring cells, which are shown in Fig. 3. If $C_{i}$ has 3 neighbors, the neighboring vertices are of the same color. On the other hand, if the neighbors are of different colors, $C_{i}$ has 2 neighbors. There exists a simple structure in triangle-free cellular network, i.e., a cell has only one neighbor, this structure can be regarded as the case in Fig. 3(b).

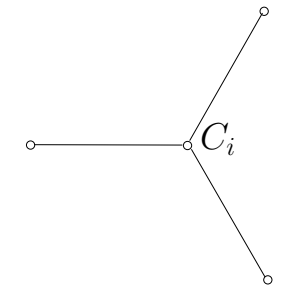

(a) Structure A: neighbors with the same base color

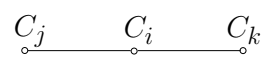

(b) Structure B: neighbors with different base colors

Figure 3: Structure of neighboring cells

For the three base colors $R, G$ and $B$, we define a cyclic order among them as $R \rightarrow G$, $G \rightarrow B$ and $B \rightarrow R$. Partition the frequency set $\{1, \ldots, \omega\}$ into three disjoint sets:

$$
F_{R}=\{1, \ldots, \omega / 3\}, F_{G}=\{\omega / 3+1, \ldots, 2 \omega / 3\}, F_{B}=\{2 \omega / 3+1, \ldots, \omega\}
$$

To be precisely, assigning frequencies from a set must in order of bottom-to-top (assigning frequencies from the lower number to the higher number) or top-to-bottom (assigning frequencies from the higher number to the lower number). Now we describe our algorithm for call control problem in triangle-free cellular networks. 
Algorithm CACO2: Handling arrival requests in a cell $C$ with color $X \in\{R, G, B\}$

1. If cell $C$ has no neighbors, just assign frequencies from 1 to $\omega$.

2. If cell $C$ has neighboring structure $A$ (Fig. 3(a)), let $Y$ be the base color of $C$ 's neighbors and $Z$ be the other third color. Assign frequency in cell $C$ as follows if no interference appear:

(a) Assign frequencies from $F_{X}$ in bottom-to-top order.

(b) If all frequencies in $F_{X}$ are used up, assign frequencies from $F_{Z}$ in bottom-to-top order if $X \rightarrow Y$; and in top-to-bottom order otherwise.

Such assignment guarantees that if $C$ uses the frequency from $F_{Z}$ after using up all frequencies from $F_{X}$, and its neighboring cell $C^{\prime}$ also uses the frequency from $F_{Z}$ after using up the frequencies from $F_{Y}, C$ and $C^{\prime}$ must assign frequency from $F_{Z}$ in different order no matter what the neighbor configuration of $C^{\prime}$ is. (This can be verified by checking this case (case 2) and the next case (case 3) of CACO2.)

3. If cell $C$ has neighboring configuration $B$ (Fig. 3(b)), let $Y$ and $Z$ be the base colors of its two neighbors, respectively. Without loss of generality, assume that $X \rightarrow Y$. Assign frequency in cell $C$ as follows if no interference appear:

(a) Assign frequencies from $F_{X}$ in bottom-to-top order.

(b) If all frequencies in $F_{X}$ are used up, assign frequencies from $F_{Y}$ in top-to-bottom order.

Theorem 7. The competitive ratio of CACO2 is at most $9 / 4$.

Proof. For a given request sequence, let $O_{i}$ and $A_{i}$ be the numbers of accepted requests in cell $C_{i}$ by the optimal offline algorithm and online algorithm CACO2, respectively. This theorem holds if $\sum_{i} O_{i} / \sum_{i} A_{i} \leq 9 / 4$. Similar to the analysis for CACO, define $B_{i}$ as the amortized number of accepted requests in cell $C_{i}$. Again, our target is to prove that $\sum_{i} B_{i} \leq \sum_{i} A_{i}$ and $O_{i} / B_{i} \leq 9 / 4$ in any cell $C_{i}$. W.l.o.g., let $X, Y$ and $Z$ denote the three colors in the network.

Intuitively, we may set $B_{i}=4 O_{i} / 9$ if $A_{i} \geq 4 O_{i} / 9$, and the remaining uncounted frequencies can be used to compensate the number of accepted frequencies in its neighboring cells. Next, we describe how to partition the remaining uncounted frequencies according to cell $C_{i}$ 's neighboring configuration. Let $H_{i j}$ be the number of frequencies used in $C_{i}$ and compensates the number of frequencies in $C_{j}$.

1. The neighboring configuration of $C_{i}$ is $A$ (Fig. 3(a)), the uncounted number of frequencies is $A_{i}-4 O_{i} / 9$, evenly distribute this number to its three neighboring cells, i.e., each neighbors $C_{j}$ of $C_{i}$ receives $H_{i j}=\left(A_{i}-4 O_{i} / 9\right) / 3$.

2. The neighboring configuration of $C_{i}$ is $B$ (Fig. 3(b)). Assume that the color of $C_{i}$ is $X$, the colors of its neighboring cells are $Y$ (cell $C_{j}$ ) and $Z$ (cell $C_{k}$ ) respectively. W.l.o.g., assume that $X \rightarrow Y, Y \rightarrow Z$ and $Z \rightarrow X$.

- If $A_{i}>\omega / 3$,

In this case, the requests in cell $C_{i}$ will use some frequencies from the top part of $F_{Y}$. 
- If $A_{j}<4 O_{j} / 9$,

there exist rejected request in $C_{j}$, thus, $A_{i}+A_{j}=2 \omega / 3$. The remaining uncounted number of frequencies in $C_{i}$ is partitioned into $\left(4 O_{j} / 9-A_{j}\right)$ and $\omega / 9$. The former part $\left(4 O_{j} / 9-A_{j}\right)$ compensates the number in $C_{j}$ (i.e., $H_{i j}=4 O_{j} / 9-$ $A_{j}$ ), and the latter part $\omega / 9$ compensates the number in $C_{k}$ (i.e., $H_{i k}=\omega / 9$ ) if $A_{k}<4 O_{k} / 9$. This compensation is justified since $4 O_{i} / 9+\left(4 O_{j} / 9-A_{j}\right)+\omega / 9=$ $4\left(O_{i}+O_{j}\right) / 9-A_{j}+\omega / 9 \leq 5 \omega / 9-A_{j}<A_{i}$.

- If $A_{j} \geq 4 O_{j} / 9$,

in this case, no compensation is needed in $C_{j}$. Let $H_{i k}=A_{i}-4 O_{i} / 9$ if $A_{k}<$ $4 O_{k} / 9$.

- If $A_{i} \leq \omega / 3$,

In this case, all frequencies used in $C_{i}$ are from $F_{X}$, and some frequencies used in $C_{k}$ may from $F_{X}$ too. If $A_{k}<4 O_{k} / 9$, all remaining uncounted number $A_{i}-4 O_{i} / 9$ compensates the number in $C_{k}$, i.e., $H_{i k}=A_{i}-4 O_{i} / 9$. No extra number of frequencies compensates the number of frequencies in $C_{j}$, i.e., $H_{i j}=0$.

We define $B_{i}$ as follows, where $H_{j i}$ is the compensation from its neighbor $C_{j}$.

$$
B_{i}= \begin{cases}4 O_{i} / 9 & \text { if } A_{i} \geq 4 O_{i} / 9 \\ A_{i}+\sum_{j} H_{j i} & \text { if } A_{i}<4 O_{i} / 9\end{cases}
$$

From previous description, we have $4 O_{i} / 9+\sum_{j} H_{i j} \leq A_{i}$ if $A_{i} \geq 4 O_{i} / 9$, thus,

$$
\begin{aligned}
\sum_{i} B_{i} & =\sum_{A_{i} \geq 4 O_{i} / 9} 4 O_{i} / 9+\sum_{A_{i}<4 O_{i} / 9}\left(A_{i}+\sum_{C_{i} \text { and } C_{j} \text { are neighbors }} H_{j i}\right) \\
& =\sum_{A_{i} \geq 4 O_{i} / 9}\left(4 O_{i} / 9+\sum_{C_{i} \text { and } C_{j} \text { are neighbors }} H_{i j}\right)+\sum_{A_{i}<4 O_{i} / 9} A_{i} \\
& \leq \sum_{A_{i} \geq 4 O_{i} / 9} A_{i}+\sum_{A_{i}<4 O_{i} / 9} A_{i} \\
& =\sum_{i} A_{i}
\end{aligned}
$$

Now we analyze the relationship between $B_{i}$ and $O_{i}$ for any cell $C_{i}$. Assuming that the color of $C_{i}$ is $X$.

1. If $A_{i} \geq 4 O_{i} / 9, B_{i}=4 O_{i} / 9$.

2. If $A_{i}<4 O_{i} / 9$,

(a) If $A_{i}<\omega / 3$

Since $A_{i}<4 O_{i} / 9$, there must exist some rejected requests in $C_{i}$. Some frequencies in $F_{X}$ are used in one of $C_{i}$ 's neighbor $C_{j}$. According to the algorithm, the neighboring structure of $C_{j}$ is $B$ (Fig. 3(b)), and $A_{i}+A_{j}=2 \omega / 3$. We say that $A_{j} \geq 4 O_{j} / 9$. Otherwise, $A_{i}+A_{j}<4 O_{i} / 9+4 O_{j} / 9=4\left(O_{i}+O_{j}\right) / 9 \leq 4 \omega / 9$, contradiction!

In this case, $H_{j i}=4 O_{i} / 9-A_{i}$, thus,

$$
B_{i}=A_{i}+\sum_{C_{k} \text { and } C_{i} \text { are neighbors }} H_{k i} \geq A_{i}+H_{j i}=4 O_{i} / 9 .
$$


(b) If $A_{i} \geq \omega / 3$ and $C_{i}$ has two neighbors $C_{j}$ with color $Y$ and $C_{k}$ with color $Z$ as shown in Fig. 3(b).

W.l.o.g., assume that $X \rightarrow Y, Y \rightarrow Z$ and $Z \rightarrow X$. According to the algorithm, after using up the frequencies in $F_{X}, C_{i}$ will use some frequencies from $F_{Y}$ until interference appear, thus, $A_{i}+A_{j} \geq 2 \omega / 3$. We claim that $A_{j}>4 O_{j} / 9$. That is because $O_{j} \leq \omega-O_{i}<\omega-9 A_{i} / 4 \leq \omega-9 \omega / 12=\omega / 4, A_{i}<4 O_{i} / 9 \leq 4 \omega / 9$, and $A_{i}+A_{j} \geq 2 \omega / 3$. Thus, we have $A_{j} \geq 2 \omega / 9>4 O_{j} / 9$.

i. If the neighboring configuration around $C_{j}$ is $A$ (Fig. 3(a)), $H_{j i}=\left(A_{j}-4 O_{j} / 9\right) / 3$, and

$$
\begin{aligned}
B_{i} & \geq A_{i}+H_{j i} \\
& =A_{i}+\left(A_{j}-4 O_{j} / 9\right) / 3 \\
& =2 A_{i} / 3+\left(A_{i}+A_{j}\right) / 3-4 O_{j} / 27 \\
& \geq 4 \omega / 9-4 O_{j} / 27 \\
& \geq 4 O_{i} / 9
\end{aligned}
$$

ii. If the neighboring configuration around $C_{j}$ is $B$ (Fig. 3(b)),

- If $A_{j} \leq \omega / 3$, we have $H_{j i}=A_{j}-4 O_{j} / 9$. Thus,

$$
B_{i} \geq A_{i}+H_{j i}=A_{i}+A_{j}-4 O_{j} / 9 \geq 2 \omega / 3-4 O_{j} / 9 \geq 4 O_{i} / 9 .
$$

- If $A_{j}>\omega / 3$, according to the description of the compensation, $H_{j i}=\omega / 9$ or $H_{j i}=A_{j}-4 O_{j} / 9$. In the former case,

$$
B_{i} \geq A_{i}+H_{j i}=A_{i}+\omega / 9 \geq \omega / 3+\omega / 9=4 \omega / 9 \geq 4 O_{i} / 9 .
$$

In the latter case,

$$
B_{i} \geq A_{i}+H_{j i}=A_{i}+A_{j}-4 O_{j} / 9 \geq 2 \omega / 3-4 O_{j} / 9 \geq 4 O_{i} / 9 .
$$

(c) If $A_{i} \geq \omega / 3$ and the neighbors of $C_{i}$ are of the same color (Fig. 3(a)),

Assume that the color of its neighboring cell is $Y$. According to the algorithm, after using up the frequencies from $F_{X}, C_{i}$ will use some frequencies from $F_{Z}$ to satisfy some requests. Since $C_{i}$ rejects some requests, we have either $A_{i}=2 \omega / 3$, or $A_{i}+A_{j}=\omega$ for some neighboring cell $C_{j}$ of $C_{i}$, which is because $C_{i}$ and $C_{j}$ assign frequencies from $F_{Z}$ in different order, and $C_{j}$ will use the frequency from $F_{Z}$ after using up the frequency from $F_{Y}$. Since $A_{i}<4 O_{i} / 9 \leq 4 \omega / 9$, the first case does not happen.

We claim that $A_{j} \geq 4 O_{j} / 9$, which is because $A_{j}=\omega-A_{i}>\omega-4 O_{i} / 9 \geq 5 \omega / 9>$ $4 O_{j} / 9$.

- If the neighboring configuration of $C_{j}$ is $A$ (Fig. 3(a)), $H_{j i}=\left(A_{j}-4 O_{j} / 9\right) / 3$. Thus,

$$
\begin{aligned}
B_{i} & \geq A_{i}+H_{j i} \\
& =A_{i}+\left(A_{j}-4 O_{j} / 9\right) / 3 \\
& =2 A_{i} / 3+\left(A_{i}+A_{j}\right) / 3-4 O_{j} / 27 \\
& \geq 5 \omega / 9-4 O_{j} / 27 \\
& >4 O_{i} / 9
\end{aligned}
$$


- If the neighboring configuration of $C_{j}$ is $B$ (Fig. 3(b)), $H_{j i}=\omega / 9$ or $A_{j}-4 O_{j} / 9$. In the former case,

$$
B_{i} \geq A_{i}+H_{j i}=A_{i}+\omega / 9 \geq 4 \omega / 9 \geq 4 O_{i} / 9 .
$$

In the latter case,

$$
B_{i} \geq A_{i}+H_{j i}=A_{i}+A_{j}-4 O_{j} / 9=\omega-4 O_{j} / 9 \geq 4 O_{i} / 9 .
$$

Combine all above cases, we have $O_{i} / B_{i} \leq 9 / 4$ in each cell $C_{i}$. Since $\sum_{i} B_{i} \leq \sum_{i} A_{i}$, we have $\sum_{i} O_{i} / \sum_{i} A_{i} \leq 9 / 4$.

Next, we prove that the lower bound of the competitive ratio for call control problem in triangle-free cellular networks is at least $5 / 3$.

Theorem 8. The competitive ratio for call control problem in triangle-free cellular network is at least $5 / 3$.

Proof. We prove the lower bound by using an adversary who sends requests according to the assignment of the online algorithm.

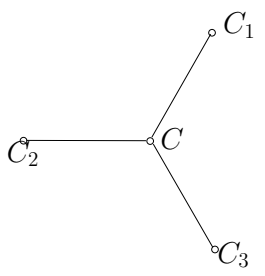

Figure 4: lower bound of competitive ratio is at least $5 / 3$

Consider the configuration shown in Figure 4.

In the first step, the adversary sends $\omega$ requests in the center cell $C$. Suppose the online algorithm accepts $x$ requests. If $x \leq 3 \omega / 5$, the adversary stop sending request. In this case, the optimal offline algorithm can accept all these $\omega$ requests, thus, the ratio is at least $5 / 3$.

If $x>3 \omega / 5$, the adversary then sends $\omega$ requests in each cell of $C_{1}, C_{2}$ and $C_{3}$. To avoid interference, the online algorithm accepts at most $\omega-x$ requests in each cell, and the total number of accepted requests is $x+3(\omega-x)=3 \omega-2 x$. In this case, the optimal offline algorithm will accept $3 \omega$ requests, i.e., reject all requests in the center cell $C$. Thus, the ratio in this case is $3 \omega /(3 \omega-2 x)$. Since $x>3 \omega / 5$, this value is at least $5 / 3$.

Combine the above two cases, the competitive ratio for call control problem in triangle-free cellular network is at least $5 / 3$.

\section{Concluding Remarks}

We have studied online call control problem in wireless communication networks and presented online algorithms in cellular networks and triangle-free cellular networks. In cellular networks, we derived an upper bound of $7 / 3$, while in triangle-free cellular networks, the upper bound and lower bound we achieved in this paper are $9 / 4$ and $5 / 3$, respectively. These bounds surpass the previous best known results. The gap between the upper and lower bound is quite big in both cases, and closing the gaps are very interesting problems for future research. 


\section{References}

[1] Ioannis Caragiannis, Christos Kaklamanis, and Evi Papaioannou. Efficient on-line frequency allocation and call control in cellular networks. Theory Comput. Syst., 35(5):521543, 2002. A preliminary version of the paper is in SPAA 2000.

[2] Ioannis Caragiannis, Christos Kaklamanis, and Evi Papaioannou. Competitive Algorithms and Lower Bounds for On-Line Randomized Call Control in Cellular Networks. Networks 52(4): 235-251, 2008. Preliminary versions are in WAOA03 and EUROPAR05.

[3] Wun-Tat Chan, Francis Y.L. Chin, Deshi Ye and Yong Zhang. Online Frequency Allocation in Cellular Networks. In Proc. of the 19th ACM Symposium on Parallelism in Algorithms and Architectures (SPAA 2007), pp. 241-249.

[4] Frédéric Havet. Channel assignment and multicoloring of the induced subgraphs of the triangular lattice. Discrete Math. 233, 219-231 (2001).

[5] V. H. MacDonald. Advanced mobile phone service: The cellular concept. Bell Systems Technical Journal, 58(1):15-41, 1979.

[6] Colin McDiarmid and Bruce Reed. Channel assignment and weighted coloring. Networks, 36(2):114-117, 2000.

[7] Lata Narayanan, and Sunil Shende. Static frequency assignment in cellular networks. Algorithmica, 29(3):396-409, 2001.

[8] Grammati E. Pantziou, George P. Pentaris, and Paul G. Spirakis Competitive Call Control in Mobile Networks. Theory of Computing Systems, 35(6): 625-639, 2002.

[9] Petra Šparl, and Janez Žerovnik. 2-local 5/4-competitive algorithm for multicoloring triangle-free hexagonal graphs. Inf. Process. Lett. 90, 239-246 (2004)

[10] Deshi Ye, Xin Han, and Guochuan Zhang. Deterministic On-line Call Control in Cellular Networks. Theor. Comput. Sci. 411(31-33): 2871-2877 (2010)

[11] Yong Zhang, Francis Y.L. Chin, and Hong Zhu. A 1-Local Asymptotic 13/9-Competitive Algorithm for Multicoloring Hexagonal Graphs. Algorithmica (2009) 54:557-567. 